\title{
Super-rotating the venusian atmosphere
}

Analysis of spacecraft observations deciphers the source of fast atmospheric rotation

Sebastien Lebonnois

Among the intriguing mysteries that remain for planetary atmospheres, the phenomenon of superrotation is still a teasing problem. An atmosphere in super-rotation rotates globally faster than the solid body of the planet. In our solar system, super-rotation is observed on Venus and the largest moon of Saturn, Titan (1). The challenge is to explain how angular momentum can accumulate in the atmosphere and what controls the atmospheric angular momentum budget. On page 405 of this issue, Horinouchi et al. (2) address this by analyzing observation data from the onboard cameras of the Venus-orbiting Akatsuki spacecraft.

The Akatsuki spacecraft has been in equatorial orbit around Venus since December 2015. Understanding the angular momentum transport in the equatorial regions is key to solving the puzzle of super-rotation. The authors retrieved several components of the angular momentum balance inthe cloud-top region, located around $70 \mathrm{~km}$ of altitude. This region is where the zonal wind speed of the venusian atmosphere is at a maximum, with values around $110 \mathrm{~m} / \mathrm{s}$ between $45^{\circ} \mathrm{N}$ and $45^{\circ} \mathrm{S}$. From a theoretical point of view, the super-rotation is based on a balance between the transport of angular momentum by the mean meridional circulation and by transient wave activity $(3,4)$. The mean meridional circulation on Venus is characterized by an equator-to-pole thermally direct circulation, transporting angular momentum upward and poleward (see the figure). The whole question is then the nature of the transients that may be the dominant players to counteract this transport. Several scenarios have been proposed, including vertical transport by eddies (such as thermal tides) and horizontal equatorward transport by planetary-scale waves. Without additional elements, answering the super-rotation question is not possible through theory alone.

General circulation models (GCMs) developed for Earth's atmosphere play a vital role in understanding atmospheric evolution. They also can be used to provide insight into other planetary atmospheres, including features not observed in Earth's atmosphere. These models are complex but extremely useful tools to explore the mechanisms taking place in the atmospheric circulation of a given planet. In the case of Venus and super-rotation, the simulations can help to decipher the relative role of each of the potential contributors to angular momentum transport (5). However, the development of realistic simulations of the atmosphere of Venus by GCMs is not an easy task. Several GCMs have finally obtained circulation and temperature structures that can fit observed fields with a reasonable agreement (6-8).

Balancing of angular momentum in the system confirms the role of transport by the mean meridional circulation and its theoretical characteristics. The models also confirm that different transient wave activities play a role in balancing this transport. In particular, thermal tides have an emphasized role in the vertical transport of angular momentum in the equatorial region. However, the wave activities that develop in these models are not always robustly established and seem to be very sensitive tomodel characteristics such as resolution, thermal forcing, or dynamical core structure (9). Therefore, as with the theory alone, this sensitivity precludes these numerical models from being fully conclusive with respect to the super-rotation mechanisms. This is where Horinouchi et al.'s analysis of the Akatsuki observational datasets becomes a key element in resolving the super-rotation mystery. The authors tracked the contrast features in the ultraviolet images of the clouds that cover the planet and analyzed heat transport using maps of brightness temperature obtained in the thermal infrared. This allowed the authors to measure or estimate horizontal and vertical transport of angular momentum by thermal tides. Other terms were also obtained: transport by horizontal Rossby waves and other transient disturbances. This strategy gives a consistent picture of the angular momentum balance at the cloud-top level, revealing a strong 
equatorward horizontal transport of angular momentum by thermal tides in the low-latitude region, mitigated by an unexpected poleward transport by horizontal turbulence.

Horinouchi et al. provide an important piece of the super-rotation puzzle that can offer a strong constraint on numerical simulations of the venusian atmosphere. However, the question of whether their analysis presents a complete picture of the angular momentum balance may still be open. The observation and analysis focus on only one level of the thick atmosphere of Venus. The possibility remains that the multiple wave activities and their impact on this very sensitive balance may be different at other levels within the 20-km-thick cloud layer. Unfortunately, investigating other levels will be very complex because of the observational challenges of such a thick atmosphere. Many viewpoints are needed to solve this type of puzzle, and the best answer to the super-rotation question will likely depend on a combination of observations, theory, and GCM simulations.

\section{References}

(1) P. L. Read, S. Lebonnois, Annu. Rev. Earth Planet. Sci. 46, 175-202 (2018)

(2) T.Horinouchi et al., Science 368, 405 (2020)

(3) P. Gierasch, J. Atmos. Sci. 32, 1038-1044 (1975)

(4) W. B. Rossow, G. P. Williams, J. Atmos. Sci. 36, 377-389 (1979)

(5) A. Sanchez-Lavega et al., Space Sci. Rev. 212, 1541-1616 (2017)

(6) S. Lebonnois, N. Sugimoto, G. Gilli, Icarus 278, 38-51 (2016)

(7) N. Sugimoto, M. Takagi, Y. Matsuda, J. Geophys. Res. Planets 119, 1950-1968 (2014)

(8) J. Mendonca, P. L. Read, Planet. \& Space Sci. 134, 1-18 (2016)

(9) S. Lebonnois et al., ISSI Scientific Report series 11, 129-156 (2013)

\section{Acknowledgements}

I thank the Centre National d'Etudes Spatiales (CNES) for support.

\section{Supercharging wind speed}

The atmosphere of Venus can circle the planet up to 60 times as fast as the rotation of the planet itself, which requires 243 Earth days. This phenomenon is called super-rotation. The angular momentum budget that causes this is a complicated combination of transport by mean meridional circulation (dark blue), thermal tides (orange), planetary-scale waves (red), and turbulent eddies (pink).

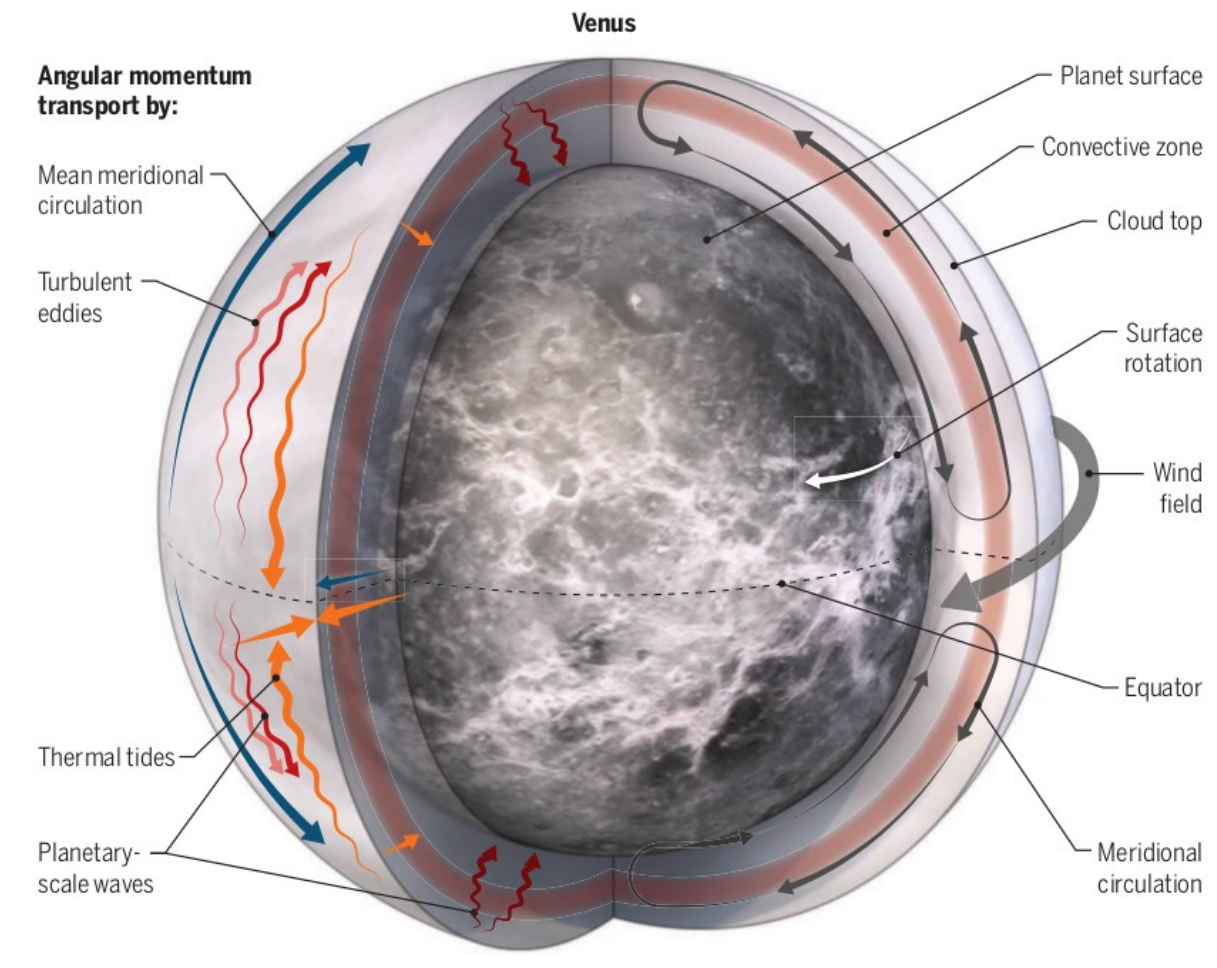

